\title{
QoS-Aware Resource Allocation and Femtocell Selection for 5G Heterogeneous Networks
}

\section{Amal Bouaziz}

Ecole Nationale d'Ingenieurs de Sfax

Ahlem Saddoud ( $\nabla$ ahlemsaddoud@gmail.com )

Universite de Sfax Faculte des Sciences Economiques et de Gestion de Sfax https://orcid.org/00000002-5314-4134

\section{lamia chaari}

ISIM Sfax: Universite de Sfax Institut Superieur d'Informatique et de Multimedia de Sfax

\section{Hakima Chaouchi}

Mines ParisTech

\section{Research Article}

Keywords: 5G, Resource Allocation, Femtocell Selection, Quality of service, Internet of things, M2M, H2H

Posted Date: June 24th, 2021

DOI: https://doi.org/10.21203/rs.3.rs-471274/v1

License: (c) (i) This work is licensed under a Creative Commons Attribution 4.0 International License. Read Full License 


\title{
QoS-Aware Resource Allocation and Femtocell Selection for 5G Heterogeneous Networks
}

\author{
${ }^{1}$ Amal BOUAZIZ, ${ }^{1}$ Ahlem SADDOUD , ${ }^{1}$ Lamia CHAARI FOURATI and \\ ${ }^{2}$ Hakima CHAOUCHI \\ ${ }^{1}$ Laboratory of Signals, Systems, Artificial Intelligence and Networks \\ (SM@RTS), Digital Research Center of SFAX (CRNS), University of SFAX, \\ TUNISIA \\ ${ }^{2}$ CNRS, SAMOVAR, Telecom Sud Paris, Institut Telecom, Paris-Saclay \\ University, Paris, France
}

\begin{abstract}
5G is not a simple cellular technology; it's a real revolution to improve the connection speed that assures Quality of Service (QoS) requirements and user satisfaction in a heterogeneous environment. 5G network is considered as a Heterogeneous Networks (HetNets) able to support a multitude of specific use cases (such as Smart Metering and Videoconferencing) and new services, where performance requirements will be extremely polarized. In this context, several key issues for $5 \mathrm{G}$ communications should be addressed to satisfy QoS provisioning. Radio resource allocation is considered as an important 5G key issue for Internet of Things (IoT) communications. In this paper, we propose a QoS-aware resource allocation and femtocell selection for 5G HetNets named QoS-RAS. Our proposed approach maximizes the total resource utilization of the network and it ensures a balanced load by selecting the suitable femtocell for each user and allocating the available resources with an adequate manner. Our work gives the best scenarios that aim to enhance system model performance in terms of resource utilization ratio, dropped request probability, total average throughput and fairness index.

Keywords: 5G, Resource Allocation, Femtocell Selection, Quality of service, Internet of things, M2M, H2H.
\end{abstract}




\section{Introduction}

Current mobile communication systems, such as the fourth generation $(4 \mathrm{G})$, are providing service regularly to the global world. Despite the great amount of data and services that are loaded in $4 \mathrm{G}$, compared to the anterior cellular network, a considerable gap persists between the human's practical needs and technologies provided by the $4 \mathrm{G}$. Therefore, the telecom industry, standards developing organization and academia have kicked off to achieve the fifthgeneration mobile network $(5 \mathrm{G})$ landmark [1]. It is hard to define $5 \mathrm{G}$ features. Nevertheless, related to current cellular networks systems, it is forecasted that 5G must have a 10 to 100 times higher number of connected user and data rate, 10 times longer battery life for low power devices and 1000 times higher mobile data volume [2]. To do so, many standardization bodies and industries are competing and spending colossal resources and efforts on evolving $5 \mathrm{G}$ researches. Some of them, such as Huawei, aimed to make $5 \mathrm{G}$ able to support massive connectivity while implementing various sets of users, services, and applications with antithetical exigencies and requirements. Others, such as LTE, were devoted to some key technologies like cloud radio, software-defined air interface, massive Multiple-Input Multiple-Output (massive MIMO) [3], etc. Therefore, we believe that $5 \mathrm{G}$ has to be an intensive network that exhibits several key technologies such as IoT (Internet of Things) and M2M (Machine-to-Machine) communications, small cell deployment, mobility management, etc.

In the following, we present the features and functionalities of IoT communications in $5 \mathrm{G}$ networks. Then, we depict the benefits of the densification concept via small cell deployment.

\subsection{IoT and M2M in the era of $5 G$}

IoT communications are considered a real evolution made by the $5 \mathrm{G}$ network to make it real. These developments represent an important issue for several

sectors of our society, particularly the economy. Organizations are present to ensure that operators meet standards for these technological developments. To 
guarantee that these issues succeed and to keep pace with the growing evolution of connected objects on the market, networks are more moving towards virtualization. The 5G introduces new architectures 44 and new features at all levels. This goes from the object itself to the applications hosted in the cloud, going through the various network layers. The uses that we make of this technology are diverse and varied. For example, the enrichment of the connected home, the autonomous vehicle, immersive videos and the arrival of medicine 2.0 and industry 4.0. The IoT is used in a wide variety of fields. It can be classified into three categories: the low-speed IoT, the high-speed IoT and the critical IoT. Uses cases range from collecting information on objects in the home, to monitor critical infrastructure. The support for Machine-to-Machine communications (M2M) is treated as one of the major troublesome technologies of $5 \mathrm{G}$ because the new generation of cellular communications will have to cope with all the requirements of $\mathrm{M} 2 \mathrm{M}$ while guaranteeing that all Human-to-Human communications $(\mathrm{H} 2 \mathrm{H})$ services are not threatened. Therefore, new emerging communications systems will need to handle with the coexistence of both types of traffic. The system of developed 5G takes into account the integration of indispensable enabling technologies for guaranteed QoS and ubiquitous connectivity to cope with to deal with the nature of particular M2M traffic. Providing connectivity to a huge number of M2M devices with their different requirements and characteristic is the main goal of $5 \mathrm{G}$. Thus, significant improvements are forecasted in forthcoming $5 \mathrm{G}$ networks, which create integrated and compatible support for M2M communications.

\subsection{Small cell Densification Concept}

Given that, 5G networks will execute applications with high requirements for data rates, reinforce network densification via small cell deployment seems to be one of the solutions to satisfy these data rate demands. Thanks to their significant ability to increase density, coverage and network capacity, it is clear why there was broad and early industry agreement that small cells will be a decisive element in $5 \mathrm{G}$ wireless networks. The advantage of small cell network 
compared to a macro-cellular network is quite reduce the number of users connected to each antenna, besides the low-cost capacity of deployment and the use of high-frequency specter bands and frequency reuse. In $5 \mathrm{G}$, small cells will also deliver new services that are based on the presence information and location of the user and/or his proximity [5]. The small cells are microcells, picocells, and femtocells. These cells are classified according to the size of the geographical area that they cover. A microcell will be deployed on a neighborhood scale, while picocells will be deployed at the scale of a large building such as a factory or a shopping center. The femtocells are deployed at the scale of a house in an apartment or company. The simultaneous operation of macro-small cells is termed heterogeneous networks (HetNets) as depicted in Fig. 1. HetNets consists of various-type cells with different wireless coverage. In HetNet, base stations of small cells [6] (i,e, Small evolved Node B (SeNB)) are located in a macrocell, knowing that they assume the same capabilities of a standard evolved Node B (eNB). Table. 1 shows the difference between the various versions of small cells. They vary in their coverage radius, output power, number of users they can handle, etc. However, all cases incorporate 3G, 4G and Wi-Fi technologies carriers.

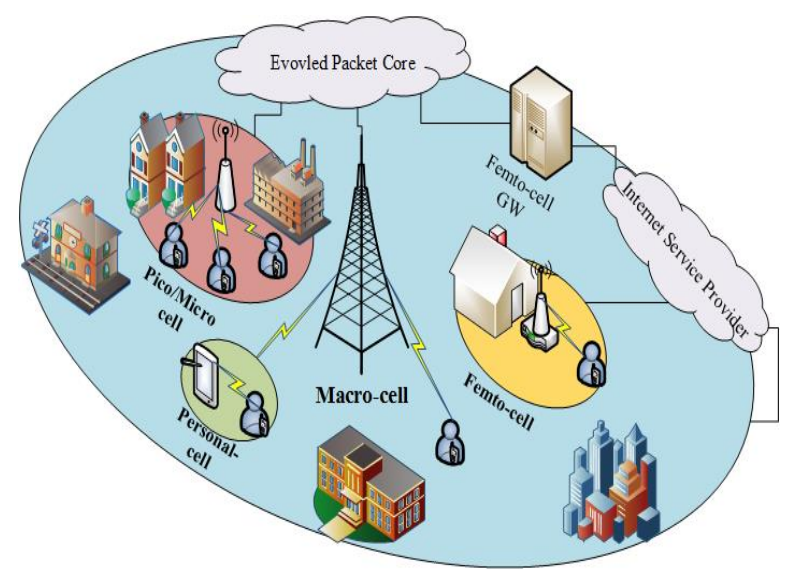

Figure 1: Heterogeneous Network Architecture. 
Table 1: Comparison among different types of cells

\begin{tabular}{|c|c|c|c|c|}
\hline & Macrocell & Microcell & Picocell & Femtocell \\
\hline Cell radius & 10 to $40 \mathrm{~km}$ & $2 \mathrm{~km}$ & $200 \mathrm{~m}$ & 10 to $50 \mathrm{~m}$ \\
\hline Indoor/Outdoor & Outdoor & Outdoor & Indoor or outdoor & Indoor \\
\hline Output power & 40 to $100 \mathrm{~W}$ & 2 to $10 \mathrm{~W}$ & $250 \mathrm{~mW}$ & 20 to $100 \mathrm{~mW}$ \\
\hline Bandwidth & 60 to $75 \mathrm{MHz}$ & 20 to $40 \mathrm{MHz}$ & $20 \mathrm{MHz}$ & $10 \mathrm{MHz}$ \\
\hline Technology & $3 \mathrm{G} / 4 \mathrm{G}$ & $3 \mathrm{G} / 4 \mathrm{G} / \mathrm{Wi}-\mathrm{Fi}$ & $3 \mathrm{G} / 4 \mathrm{G} / \mathrm{Wi}-\mathrm{Fi}$ & $3 \mathrm{G} / 4 \mathrm{G} / \mathrm{Wi}-\mathrm{Fi}$ \\
\hline Cost & $60.000 \$ /$ year & $30.000 \$ /$ year & $10.000 \$ /$ year & $200 \$ /$ year \\
\hline $\begin{array}{c}\text { Power } \\
\text { Consumption }\end{array}$ & High & Moderate & Low & Low \\
\hline
\end{tabular}

\subsection{Related Works}

In the purpose to enhance $5 \mathrm{G}$ network functionalities and to guarantee QoS requirements for users, several contributions are proposed in the literature. We classify these related works into two categories including resource allocation and cell selection in 5G HetNets.

\subsubsection{Related works of resource allocation in $5 G$ HetNets}

In [10, the authors proposed two schedulers for IoT communications based on QoS requirements of $\mathrm{M} 2 \mathrm{M}$ and $\mathrm{H} 2 \mathrm{H}$ flows by guaranteeing network performance and avoiding ineffective exploitation of available resources. The first one is a static scheduler that presents an allocation strategy of available resource blocks (in the eNB) between users at one TTI [11. The second scheduler, named Dynamic Borrowing Scheduler (DBS), presents an extended version of the first scheduler using a borrowing policy for resource block allocation in the purpose to decrease the percentage of flow rejection and to maximize the bandwidth utilization rate [12. In [13, the authors propose a resource allocation scheme and dynamic power control for the next generation cellular networks $(5 \mathrm{G})$. The objective is to mitigate the resource reuse interference in a multi-cell network between D2D user equipment's (DUEs) and cellular user equipment's (CUEs). In addition, the authors propose in [14] a resource allocation scheme for cooperative hybrid $\mathrm{FSO} / \mathrm{mmW}$ 5G fronthaul network to optimize network 
reliability, average transmitted power and average BER. The proposed scheme is considered as a discrete linear multi-objective optimization problem (ILP-MOP) achieving better performance at all weather conditions. In [15], a resource allocation scheme based on a genetic algorithm (GA) is proposed for $5 \mathrm{G}$ networks. In this scheme, a resource is allocated to those D2D pairs who create less interference. In [16, the authors propose a novel resource control algorithm based on long short-term memory for the 5G ultra-dense network. The proposed model makes localized prediction of future traffic characteristics such as future buffer occupancy status forecasting probable congestion.

\subsubsection{Related works of cell selection in $5 G$ HetNet}

In [17, a cell selection and user association method are proposed for 5G heterogeneous networks using Bayesian game. The objective is to maximize the probability of proper association and to enhance the QoS performance in terms of achieved latency. Although this method could be efficient in achieving low latency objective the packet loss probability and its impact on the system performance are neglected in [17. In [18, an optimal base station selection is proposed for smart factories based on two metrics the maximum SINR (Signal to Interference plus Noise Ratio) and the maximum receive power. Experimental results prove that the maximum receive power is an optimal technique for base station selection for smart factories. However, 18 neglects different classes of traffic supported by $5 \mathrm{G}$ network. Authors proposed, in [19, an optimal cell selection method when many higher frequencies are layered. Only the system throughput is well improved in a blocker deployment environment. In order to unload traffic to light load, D2D (Device-to-Device) serves as the edge computing center. A joint relay selection method is proposed in 20 based on this model to offload macro-cell users to small cell MEC (Mobile Edge Computing) application servers. Furthermore, dual connectivity is introduced to manage user mobility and network access in the small cells. Authors exploit dual connectivity in 21] for throughput maximization, multihop routing from small to the macro cell, and selection of a small cell eNB for user equipment (UE). 


\subsection{Motivations and objectives of this paper}

From related works, the most contributions introduce separately resource allocation mechanism and cell selection for 5G HetNets. Moreover, the existing solutions need to be reviewed with the expansion of IoT communications in wireless systems to put good use of the technology. For these reasons, we propose a QoS-aware resource allocation and cell selection for $5 \mathrm{G}$ HetNets, named QoSRAS. Our proposed approach introduces a joint solution for resource allocation and femtocell selection. The primary contributions are to perform a resource allocation and a femtocell selection with the objective to 1) maximize the total resource utilization, 2) ensure a balanced load by selecting the suitable femtocell for each user, 3) fairly allocate the available resources, and to 4) enhance the total average throughput for $5 \mathrm{G}$ specific use cases (such as Smart Metering and Videoconferencing).

The rest of this paper is organized as follows. In the next section, a description of the system model is provided. Proposed QoS-RAS scheme is detailed in section III. Then, performance analysis and comparison scenarios are presented in section IV. Finally, the conclusion and future works are drawn in Section V.

\section{System Model}

\subsection{Network Architecture}

Our network architecture is brought out from a similar model adopted in past works [22, with appropriate modifications in order to be applied in different use cases. In this work, we consider the downlink (DL) data transmission of two-tiered cellular network, where one macrocell coverage is underlaid with femtocells as depicted in Fig. 2. The DL signaling is assumed to use Orthogonal Frequency Division Multiple Access (OFDMA). The Physical Resource Block (PRB) is the basic element in DL direction of 5G LTE. They are allocated to each user equipment (UE) according to its traffic type, demand and QoS requirements. The PRB is defined in both the time and frequency domain. On one hand, the time-domain consists of a single time slot $(0.5 \mathrm{~ms})$ including 7 
symbols of OFDMA. On the other hand, the frequency domain involves 12 adjacent subcarriers of $15 \mathrm{kHz}$ each resulting in a total PRB bandwidth of $180 \mathrm{kHz}$. The whole bandwidth of the network is presumed to be divided in orthogonal subchannels. Each one is assumed that can be allocated to one single user at the same time to avoid inter-channel interference between user groups. Besides, in order to minimize interference, contiguous stations are attributed to different channel groups. The range of area is entirely covering by a single macro base station (BS) eNB $M$ located at the center of the cell (0.0). In 22, femtocells were uniformly located in the cell. In the current environment, we have a set of femtocells $i$, with $i \in\{1, \ldots, F\}$. We assume $F=12$ is the total number of femtocells randomly and arbitrarily deployed in the macrocell coverage forming and overlay layer. The Home eNB (HeNB) are located at $\left(x_{i}, y_{i}\right)$. We note HeNB is the name of femtocell base station. Each BS (either the eNB or HeNB) handles a set of UEs $u \in\{1, \ldots, U\}$ who are evenly distributed in the geographical area. Two types of user, H2H users and M2M users, are considered. We assume that UEs $n \in\{1, \ldots, N\}$ with $N$ is the total number of M2M users and a set of UEs $h \in\{1, \ldots, H\}$ with $H$ is the total number of $\mathrm{H} 2 \mathrm{H}$ users at one Transmission Time Interval (TTI). Furthermore, the number of the accessing user follows the Poisson point process with a density of $\lambda$. The network is assumed that will work in an extremely backlogged system where all stations supply Continuous Bit Rate (CBR) applications to their associated UEs to fully use the allocated

bandwidth. As well as, the network is assumed to be static when there are no departures of present demands or arrivals of new ones. Useful notations are listed in Table 2 for the best understanding of the proposed approach.

\subsection{Problem Formulation}

In this section, we give an optimization model to find the optimal solution of resource allocation and femtocell selection, with the available resources of each station (eNB or HeNB). These resources are shared by different existing traffic types. The problem formulation deals with both $\mathrm{M} 2 \mathrm{M}$ and $\mathrm{H} 2 \mathrm{H}$ users. Our intention is 1) to maximize the resource utilization ratio within the system 
Table 2: Notations

\begin{tabular}{|c|c|}
\hline Notations & Descriptions \\
\hline$\alpha$ & value between 0.5 and 0.9 \\
\hline$\beta$ & value between 0.5 and 0.9 \\
\hline$N$ & The total number of M2M users \\
\hline$H$ & The total number of $\mathrm{H} 2 \mathrm{H}$ users \\
\hline$F$ & The total number of femtocells deployed in the macrocell \\
\hline$C_{n, i}$ & The charge factor of a femtocell $i$ for user $n$ \\
\hline$G_{n, i}$ & The channel gain between a user $n$ and a femtocell $i$ \\
\hline$W_{n, i}$ & The utility function \\
\hline$r_{n, i}$ & The instantaneous end-to-end from femtocell $F_{i}$ to user $n$ \\
\hline$\overline{r_{n}}$ & The average of all end-to-end rates offered by neighboring HeNB \\
\hline$R S_{M}$ & Static resources of macrocell $M$ \\
\hline$R D_{M}$ & Dynamic resources of macrocell $M$ \\
\hline$R G_{M}$ & Global resources of macrocell $M$ \\
\hline$R S_{i}$ & Static resources of femtocell $i$ \\
\hline$R D_{i}$ & Dynamic resources of femtocell $i$ \\
\hline$R G_{i}$ & Global resources of femtocell $i$ \\
\hline$R A_{i, x}$ & Allocated resources of femtocell to user $x i$ \\
\hline$R A_{M, y}$ & Allocated resources of Macrocell $M$ to user $y$ \\
\hline$X$ & Number of users served by HeNB \\
\hline$Y$ & Number of users served by eNB \\
\hline$x$ & index of users served by HeNB $x \in\{1, . ., \mathrm{X}\}$ \\
\hline$y$ & index of users served by eNB $y \in\{1, . ., Y\}$ \\
\hline$i$ & index of femtocells $i \in\{1, \ldots, \mathrm{F}\}$ \\
\hline
\end{tabular}




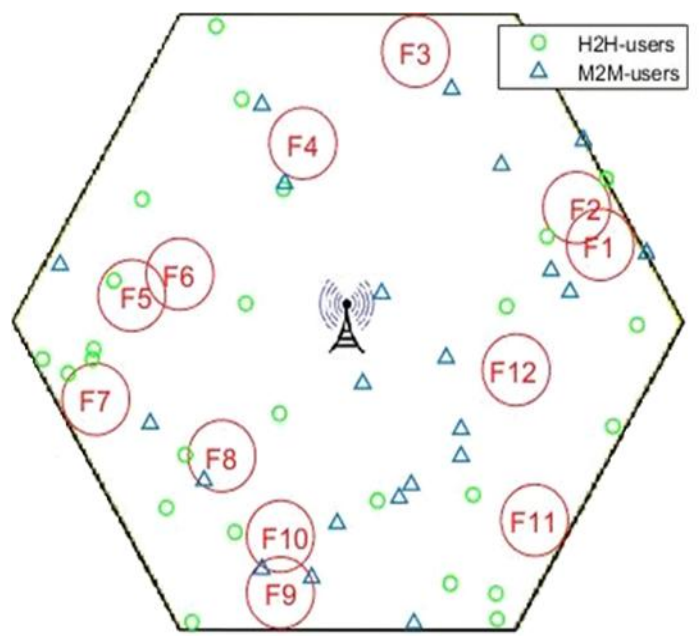

Figure 2: Network Model Scheme.

and 2) to select the suitable femtocell with the maximum value of $W_{n, i}$. Thus the model has two-objective optimization problem. The objective function is summarized in Equation 1 as follows:

$$
\operatorname{Max}\left\{\begin{array}{c}
W_{n, i}=a \cdot C_{n, i}+b \cdot R_{n, i}+\text { c. } G_{n, i} \\
R U=\frac{1}{R G}\left(\sum_{i=1}^{F} \sum_{x=1}^{X} R A_{i, x}+\sum_{y=1}^{Y} R A_{M y}\right)
\end{array}\right.
$$

Our purpose is to maximize two-objective function. The first objective intents to maximize the utility function $W_{n, i}$ and to select the appropriate femtocell for M2M users and the second aims to maximize the resource utilization function $R U$.

Firstly, the maximization of the utility function for femtocell selection is presented by equation 2 .

$$
\operatorname{Max} W_{n, i}=a . C_{n, i}+b \cdot R_{n, i}+c . G_{n, i},
$$

subject to

$$
\begin{gathered}
C_{n, i}=\frac{R G_{i}-R A_{i}}{R G_{i}}, \\
R A_{i}=\sum_{x=1}^{X} R A_{i, x} ; \forall x \in\{1, \ldots, X\}
\end{gathered}
$$




$$
\begin{gathered}
R_{n, i}=\frac{r_{n, i}}{\overline{r_{n}}}, \\
G_{n, i}=10^{-P L_{n, i} / 10}, \\
a+b+c=1,
\end{gathered}
$$

The utility function is a linear combination of three factors and it is calculated according to the following constraints. The constraint (3) ensures that the charge factor $C_{n, i}$ of femtocell $i$, is defined as the ratio of the number of available radio resources at the HeNB to its total capacity. These available resources at the HeNB are expressed by equation (4) as the total resources assigned to all served users in the femtocell $i$. Moreover, equation (5) ensures that the rate factor $R_{n, i}$ is the instantaneous rate factor offered by each femtocell $i$ to user $n$ divided by the mean data rate provided by the nearby $i$ during one TTI. The channel gain $G_{n, i}$ between the user and the candidate femtocell $i$ is also taken into consideration. It is expressed by equation 6 where $P L_{n, i}$ denotes the pathloss between the femtocell $i$ and the user $n$ (detailed in our previous work [22]). Finally, equation (7) ensures that the sum of the weights a, b and c is equal to 1.

Secondly, our target is to maximize the resource utilization function $R U$ defined by equation 8 .

$$
\operatorname{Max} R U=\frac{1}{R G}\left(\sum_{i=1}^{F} \sum_{x=1}^{X} R A_{i, x}+\sum_{y=1}^{Y} R A_{M y}\right)
$$

subject to

$$
\begin{gathered}
R G=\sum_{i=1}^{F}\left(R G_{i}\right)+R G_{M} ; \forall i \in\{1, \ldots, F\} \\
R G_{i}=\alpha R S_{i}+(1-\alpha) R D_{i} \\
R G_{M}=\beta R S_{M}+(1-\beta) R D_{M}
\end{gathered}
$$




$$
\begin{aligned}
& \sum_{x=1}^{X} R A_{i, x} \leq R G_{i} ; \forall x \in\{1, \ldots, X\} \\
& \sum_{y=1}^{Y} R A_{M, y} \leq R G_{M} ; \forall y \in\{1, \ldots, Y\}
\end{aligned}
$$

As it is mentioned above, the function $R U$ is the resource utilization ratio that computes the rate of the allocated resources to the global ones at one TTI. $R G$ denotes the global resources in the system, however, $R A_{i, x}$, (respectively $R A_{M, y}$ ) represents the allocated resources for user $x$ in femtocell $i$, (respectively macrocell $M) . X$ is the total number of served users by the HeNB of femtocells and $Y$ is the total number of served users by the macrocell $M$. So as to solve the objective function stated by eq (1), a set of constraints are needed. The constraint $(9)$ ensures that $R G$ is the sum of the global resources of all femtocells $R G_{i}$ (expressed in the constraint 10 ), plus the global resources of the macrocell $R G_{M}$ (expressed in the constraint (11)). As we explained above, the global resource of each station $i$, (respectively $M$ ) is the sum of static resources $R S_{i}$, (respectively $R S_{M}$ ) and dynamic resources $R D_{i}$, (respectively $R D_{M}$ ). The division is done randomly with respect to the random variables $\alpha$ and $\beta$, where

$0.5 \leq \alpha, \beta \leq 0.9$. Besides, in the constraints 12 and 13 , we consider that the upper limit of allocated resource in each cell is equal to the global one.

\section{Proposed femtocell selection and QoS-aware resource allocation Scheme}

In order to solve the problem (1) detailed previously, we propose a joint QoS-aware resource allocation and a femtocell selection scheme named QoSRAS. In fact, with the limited available resources of the system, it is necessary to manage an efficient resource allocation method that intends to maximize the resource utilization within the system and to ensure a balanced load by selecting the adequate femtocell for users. Accordingly, a description of the proposed approach with its two stages is given and illustrated in Fig.3. The 
first stage presents a selection of the suitable femtocell with a maximum value of the utility function. Jointly, the second stage gives a QoS-aware resource allocation in order to serve M2M and $\mathrm{H} 2 \mathrm{H}$ requests. Our QoS-RAS ensures the selection of the relevant femtocell $i$ by computing the utility function in the first stage. It provides also a resource allocation strategy between $\mathrm{H} 2 \mathrm{H}$ and $\mathrm{M} 2 \mathrm{M}$ users.

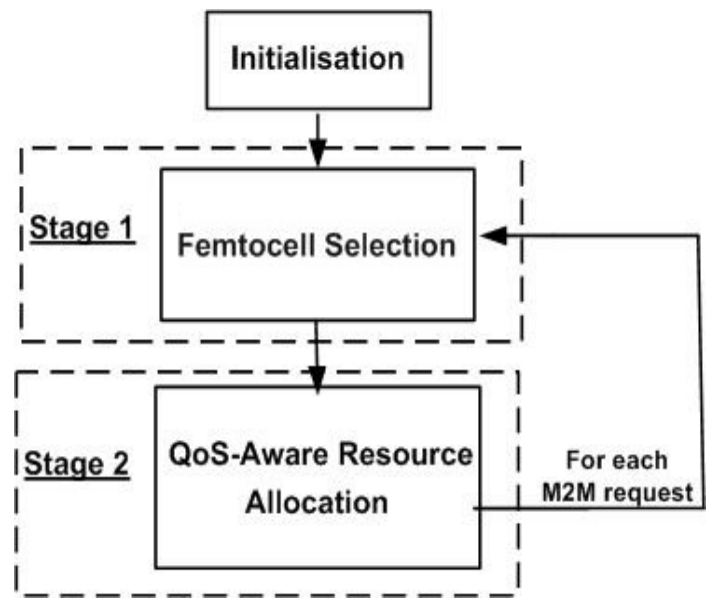

Figure 3: Proposed Resource Allocation Scheme.

After the initialisation step, the utility function process is launched in order to select the suitable femtocell for each M2M request. This function is calculated for each user $n$ from the appropriate HeNB according to equation 2 . Starting from the fact that such station will be selected only if it acquires enough resources to serve either $\mathrm{M} 2 \mathrm{M}$ or $\mathrm{H} 2 \mathrm{H}$ demands, the utility function is introduced to select the best femtocell and to satisfy M2M user demands. This utility function denoted by $W_{n, i}$, provides the suitable femtocell $i$ for each user $n$ and it takes into consideration three parameters: the charge factor $C_{n, i}$ defined by equation 3 , the rate factor $R_{n, i}$ given by equation 5 and the channel gain $G_{n, i}$ between a user $n$ and the femtocell $i$ defined previously by equation 6. The flowchart illustrated in figure 4 gives a description of the utility function.

The next stage has to do with the QoS-aware resource allocation model. 


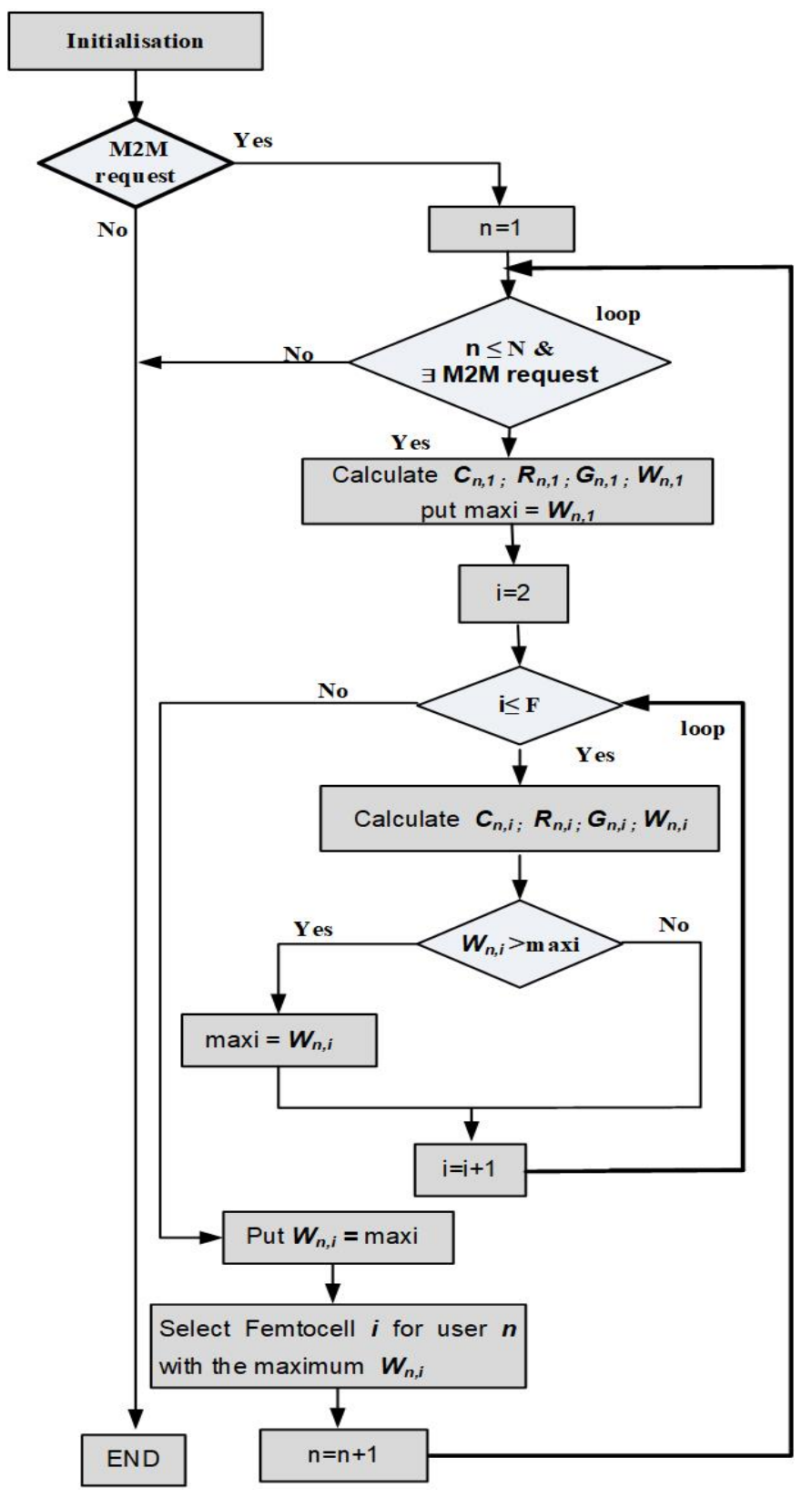

Figure 4: Utility Function Flowchart.

As it mentioned in the previous section, we propose a dynamic model where the resources of each station (either eNB or HeNB) are divided into static and 
dynamic resources. If there are enough available resources to supply service to users, the static resources will be used preferably. Whereas, when it is about large system traffic, the dynamic resources will be allocated according to the type of the users. We take into consideration the QoS requirements of users in the resource allocation strategy in order to support two specific use cases: smart metering and videoconferencing, which are detailed below.

\subsection{Smart Metering Scenario}

In order to guarantee QoS requirements for smart metering applications, we prioritize M2M requests over $\mathrm{H} 2 \mathrm{H}$ ones. The corresponding resource allocation scenario is illustrated in Fig 5 . After the execution of the utility function process, M2M request has the ability to be accepted :

- Case 1: if there are enough static resources $R S_{i}$ in the selected femtocell $i$

- Case 2: If case 1 is not satisfied, we check the availability of the dynamic resources $R D_{i}$ of the appropriate femtocell.

- Case 3: If case 2 is not satisfied, the system makes use of the static resources $R S_{M}$ of the macrocell $M$.

- Case 4: If case 3 is not satisfied, the M2M request will be served from the dynamic resources $R D_{M}$ of the macrocell $M$.

Therefore, the system will abort the M2M request when resources are fully allocated.

According to the $\mathrm{H} 2 \mathrm{H}$ request, we check the availability of static resources $R S_{M}$ of the macrocell $M$ firstly. If there are not enough resources to satisfy $\mathrm{H} 2 \mathrm{H}$ request, the system verifies the availability of the dynamic resource $R D_{M}$ of the macrocell. If not, the system makes use of the dynamic resources of the selected femtocell $R D_{i}$ with a maximum value of the SINR. Otherwise, the $\mathrm{H} 2 \mathrm{H}$ request will be rejected. In this scenario, the M2M requests can be served through several cases and so they have more chance to be accepted than $\mathrm{H} 2 \mathrm{H}$ 


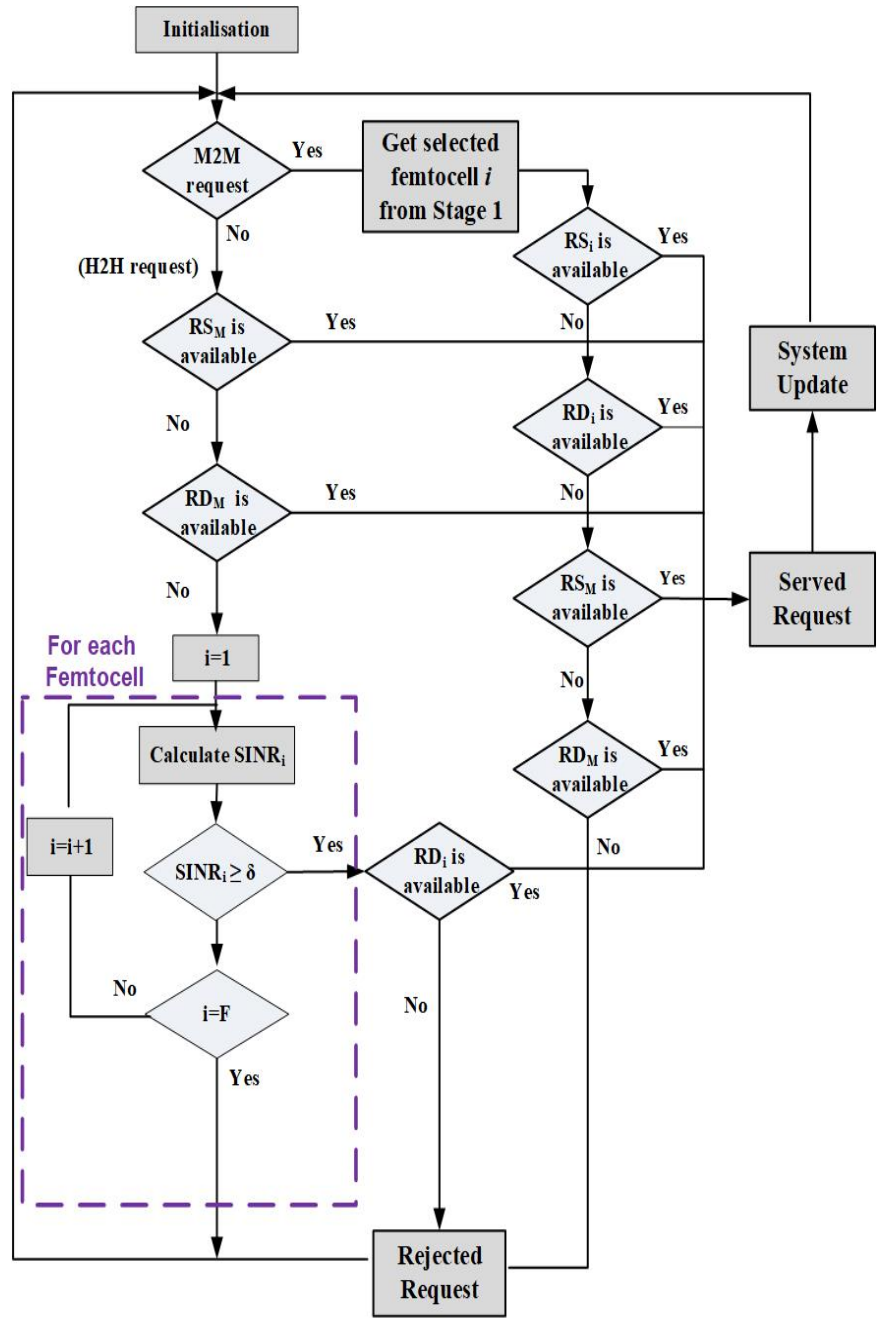

Figure 5: Resource Allocation Flowchart for Smart Metering Scenario.

demands. Furthermore, our model gives QoS satisfaction to the smart metering application as it enhances the acceptance probability of M2M users.

\subsection{Videoconferencing Scenario}

In the videoconferencing scenario, illustrated in Fig 6 , H2H demands are more prioritized than $\mathrm{M} 2 \mathrm{M}$ requests. $\mathrm{H} 2 \mathrm{H}$ users can be served according these cases: 
- Case 1: there are enough static resources $R S_{M}$ in the macrocell $M$.

- Case 2: the dynamic resources $R D_{M}$ of the macrocell $M$ are available to satisfy the request.

- Case 3: the system makes use of the dynamic resources of the selected $R D_{i}$ femtocell with a maximum value of the SINR.

Otherwise, the $\mathrm{H} 2 \mathrm{H}$ demand will be rejected when all resources are fully allocated. According to the M2M requests, the system verifies the static resources $R S_{i}$ of the selected femtocell $i$ (from the result of the utility function $W_{n, i}$ ) firstly. Elseways, the M2M demands can be served from the available dynamic resources $R D_{M}$ in the macrocell. Correspondingly, the system offers more flexibility for $\mathrm{H} 2 \mathrm{H}$ communications to be accepted and so to enhance $\mathrm{QoS}$ of the videoconferencing applications.

\subsection{Performance Metrics}

Then, we define several important metrics for evaluations and comparisons, including packet dropping probability, system throughput, SINR (Signal to Interference plus Noise Ratio), and Fairness index. Meanwhile the impact factors considered in this work include various numbers of UEs. First of performance metric is Request Dropped Probability (RDP) in the whole system, defined by:

$$
R D P=\frac{\sum_{u=1}^{U} R_{u}^{\text {dropped }}}{\sum_{u=1}^{U} R_{u}^{\text {total }}},
$$

where $R_{u}^{\text {dropped }}$ represents a dropped request of a user $u$ and $R_{u}^{\text {total }}$ denotes the total number of traffic of a user $u$. Lower the $R D P$ is corresponding to a higher performance.

Second of performance metric is throughput. The practical capacity of user $u$ can served by either the eNB or HeNB is defined as:

$$
C_{M / i, u}=\Delta f \times \log _{2}\left(1+\alpha S I N R_{M / i, u}\right)
$$




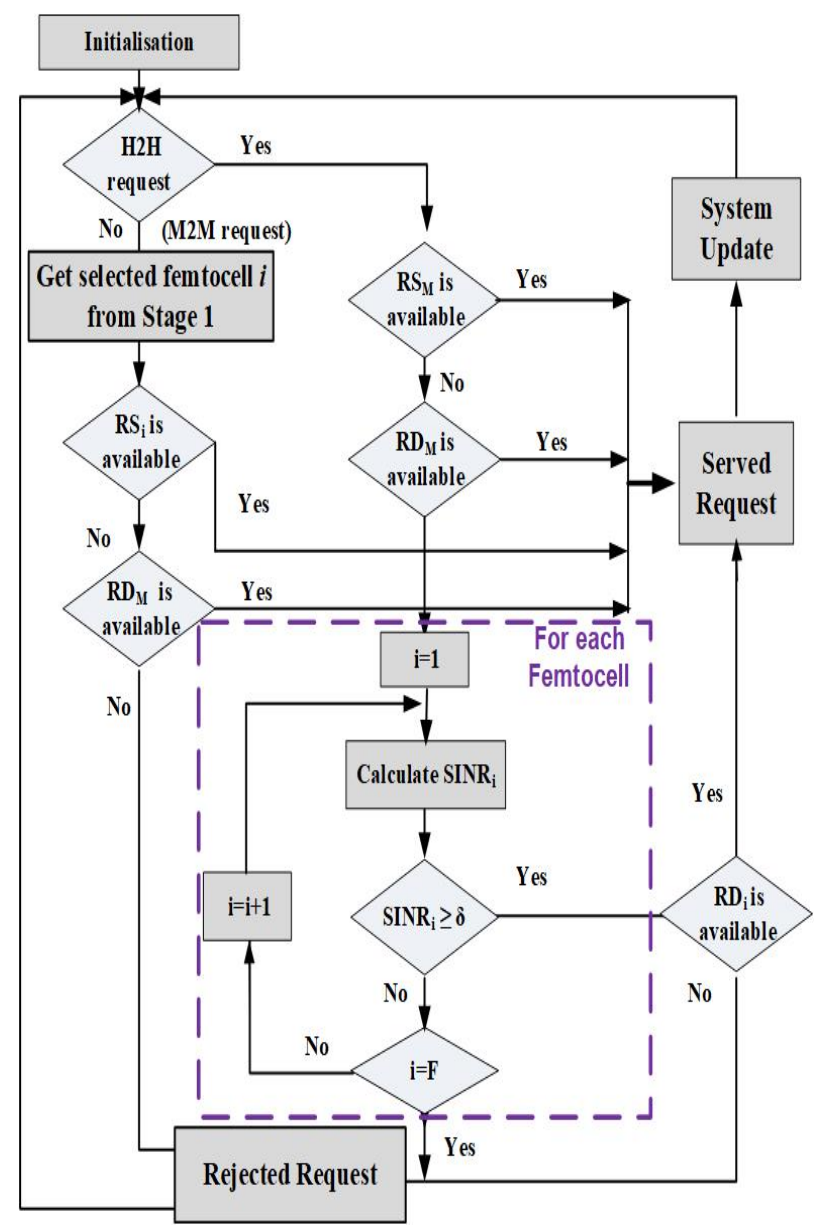

Figure 6: Resource allocation Flowchart for Videoconferencing Scenario.

where, $\Delta f$ is the subcarrier spacing and $\alpha$ is a constant for target Bit Error Rate (BER), which defined by $\alpha=-1.5 / \ln (5 B E R) . S I N R_{(M / i, u)}$ is the estimation of the received $S I N R$ of user $u$, when the user is interfered from adjacent macrocells and all the contiguous femtocells. In our analysis, the SINR calculation is expressed by the equation 16

$$
\operatorname{SIN} R_{M / i, u}=\frac{P_{M / i, u} G_{M / i, u}}{N_{0} \Delta f+\sum_{M^{\prime} \neq M} P_{M^{\prime}, u} G_{M^{\prime}, u}+\sum_{i^{\prime} \neq i} P_{i^{\prime}, u} G_{i^{\prime}, u}},
$$


where $P_{M / i, u}$ is the transmitted power of serving eNB $M$ or HeNB $i . G_{M / i, u}$ represents the channel gain of UE $u$ receiving power transmitted by eNB $M$ or HeNB $i . \quad P_{M^{\prime}, u}$ and $P_{i^{\prime}, u}$ are the transmitted power of neighbouring eNB $M^{\prime}$ and HeNB $i^{\prime}$, respectively. Similarly, $G_{M^{\prime}, u}$ (respectively $G_{i^{\prime}, u}$ ) denotes the channel gain between a UE $u$ and neighbouring eNB $M^{\prime}$ (respectively HeNB $i^{\prime}$ ). $N_{0}$ represents the white noise spectral density.

Finally, the overall throughput of serving can be expressed as follows:

$$
T h_{M / i, u}=\sum_{M / i} \sum_{u} \beta_{M / i, u} C_{M / i, u}
$$

where, $\beta_{M / i, u}$ represents the subcarrier assignment for macrocell (respectively femtocell) users $u$. When the subcarrier is assigned to user, $\beta_{M / i, u}=1$. Otherwise, $\beta_{M / i, u}=0$.

The last one is the Jain's fairness index. It measures the level of satisfaction of the different users in the system. In fact, we focus on the performance of the proposed model with regards to the throughput.

$$
F I=\frac{\left(\sum_{u}^{U} T h_{M / i, u}\right)^{2}}{U \sum_{u}^{U} T h_{M / i, u}^{2}}
$$

\section{Performance analysis and comparison}

We focus in this section on the performance evaluation of our proposed method QoS-RAS provision in 5G heterogeneous network. In order to validate our proposal, our study is carried out and the simulations are performed using MATLAB. We give the main parameters of the simulation model in Table 3.

\subsection{Smart Metering Scenario Simulation}

Performance evaluation of smart metering scenario is given by Fig 7 , Fig 8 and Fig 9. In Fig 7, we compute the dropped request probability vs. the number of users ( $\mathrm{H} 2 \mathrm{H}$ and $\mathrm{M} 2 \mathrm{M})$. Through this figure, we prove that the dropped request probability for macrocell-only scenario is almost $60 \%$ higher than the dropped 
Table 3: Simulation Parameters

\begin{tabular}{|l|l|}
\hline Parameters & Values \\
\hline Macrocell radius $(\mathrm{m})$ & 250 \\
\hline Femtocell radius $(\mathrm{m})$ & 20 \\
\hline Frequency $(\mathrm{GHz})$ & 2 \\
\hline eNB power $(\mathrm{dBm})$ & 46 \\
\hline HeNB power $(\mathrm{dBm})$ & 20 \\
\hline Outdoor walls loss low $(\mathrm{dB})$ & 20 \\
\hline Indoor walls loss liw $(\mathrm{dB})$ & 5 \\
\hline Channel bandwidth $(\mathrm{MHz})$ & 10 \\
\hline Modulation scheme & $16 \mathrm{QAM}$ \\
\hline Sub-carrier spacing $(\mathrm{KHz})$ & 15 \\
\hline White noise $(\mathrm{dBm} / \mathrm{Hz})$ & -174 \\
\hline
\end{tabular}

request probability for $\mathrm{M} 2 \mathrm{M}$ users and $25 \%$ than the ones of $\mathrm{H} 2 \mathrm{H}$ dropped request probability. The fact that $\mathrm{M} 2 \mathrm{M}$ users are more prioritized than $\mathrm{H} 2 \mathrm{H}$ ones is highlighted in this figure for the different values of $\alpha$ and $\beta$. Indeed, the M2M minimal dropped request probability is achieved when $\alpha=0.9$ and $\beta=0.9$ since $\mathrm{H} 2 \mathrm{H}$ demands are denied to be served by the static resources of femtocells. Fig 8 illustrates the improvement of fairness among users after the deployment of the femtocells, mainly if $\alpha=0.5$. In addition, the fairness index exceeds $90 \%$ which confirms that the utility function satisfies all users in the system. Fig 9 illustrates the CDF of the total user throughput which is affected by the total number of users in the system. As it is shown in this figure, the CDF of the total user throughput increases as the user throughput increases.

\subsection{Videoconferencing Scenario Simulation}

The videoconferencing scenario is evaluated in terms of dropped probability of request (Fig 10), fairness index (Fig 11) and resource utilization rate (Fig 12). In Fig 10, we compute the dropped request probability vs. the number of total users (H2H and M2M). Since we give more priority to $\mathrm{H} 2 \mathrm{H}$ requests to ensure QoS requirement for videoconferencing application, M2M demands are denied to be served by femtocells resources. This graph proves that the minimal dropped request probability for $\mathrm{H} 2 \mathrm{H}$ requests is achieved when $\alpha=0.9$ and $\beta=0.9$. 


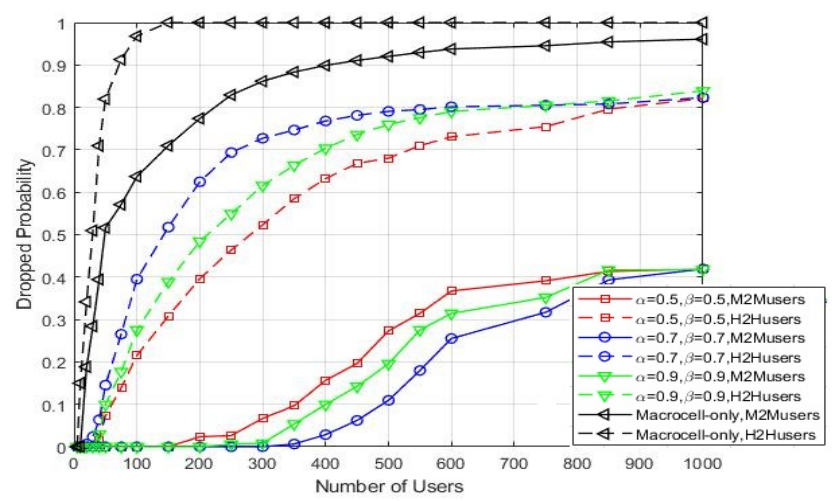

Figure 7: Dropped Probability with different values of $\alpha$ and $\beta$.

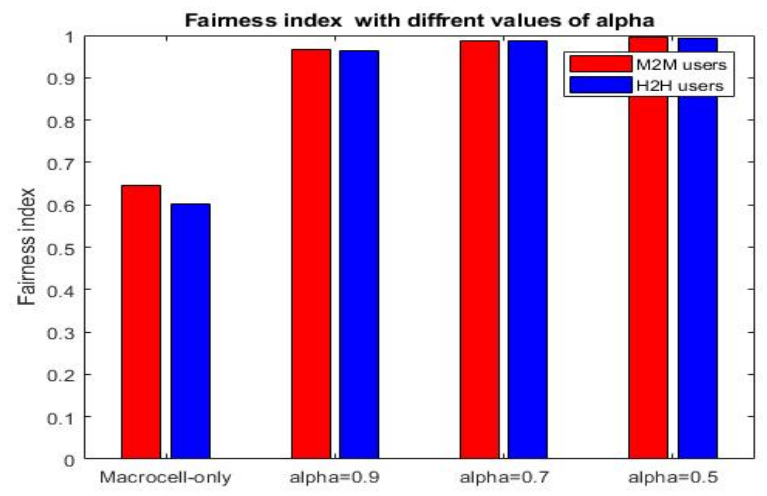

Figure 8: Fairness index per type of request with different values of $\alpha$

We notice that the best scenario for $\mathrm{H} 2 \mathrm{H}$ users is achieved when $\alpha=0.5$ since they shared half of the resources of the femtocells. According to macrocellonly scenario, the dropped request probability for M2M requests is higher than the dropped request probability for $\mathrm{H} 2 \mathrm{H}$ users. Through Fig 11 , the fairness index is depicted for different types of users for both macrocell-only scenario and for different values of $\alpha$. We notice the enhancement of this index with the deployment of femtocells in our proposed QoS-RAS and mainly for $\mathrm{H} 2 \mathrm{H}$ users. Moreover, we observe that the fairness index among users reaches its maximum and surpasses $90 \%$ when $\alpha=0.5$. In Fig, 12 we compute the rate 


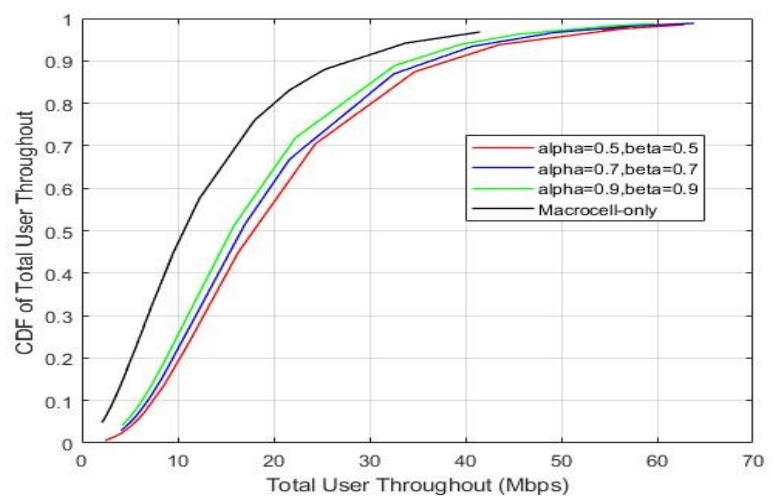

Figure 9: CDF of the total user throughput

of the resource utilization in the system as the number of total users. In the scenario of macrocell-only, the resources are almost fully allocated from 170 users. According to the rest of the scenarios, the resources are totally allocated from 1000 users. Even on the side of HeNB, the resources can be shared between both traffic types and so there is not any waste of resources.

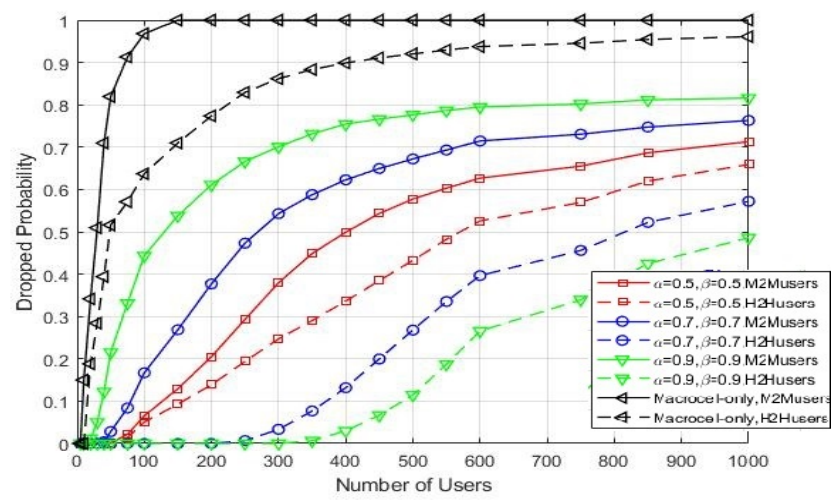

Figure 10: Dropped request H2H priority

\subsection{Comparison}

As it mentioned before, authors has proposed in [10, a Dynamic Borrowing Scheduler (DBS) for M2M and $\mathrm{H} 2 \mathrm{H}$ flows based on QoS requirements. DBS 


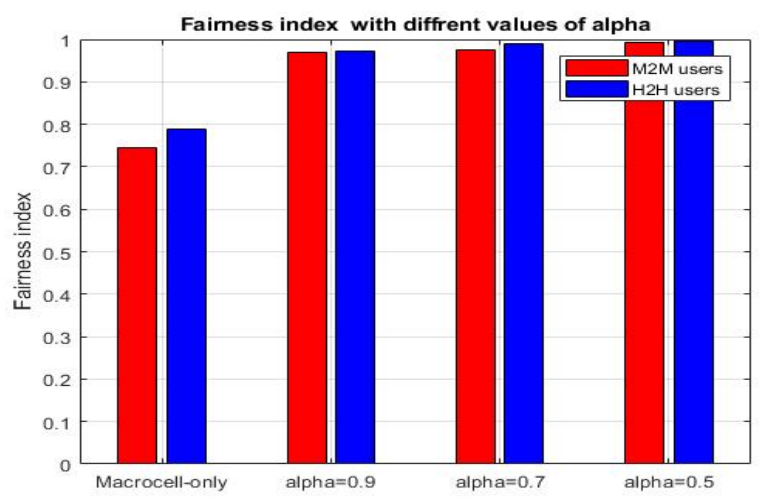

Figure 11: Fairness index

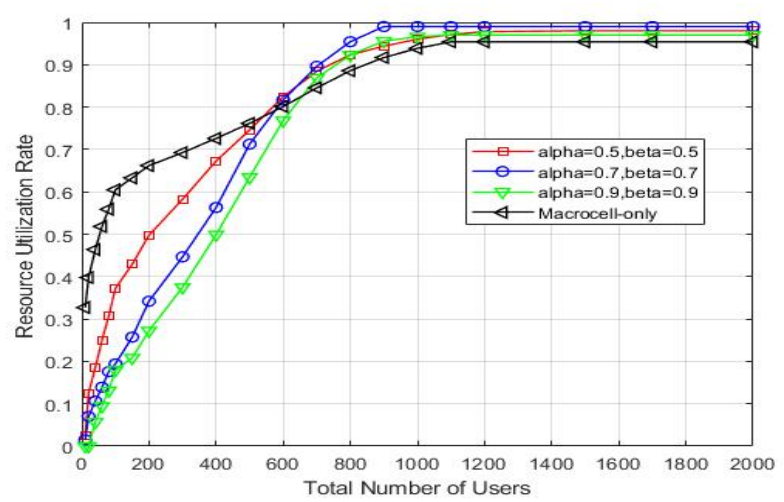

Figure 12: Resource utilization rate $\mathrm{H} 2 \mathrm{H}$ priority

uses a borrowing policy for resource blocks allocation in order to decrease the dropping probability of flows and to maximize the bandwidth utilization rate. Since our proposed algorithm Qos-RAS has the same objectives, a comparison between those two approaches is done in this section. This comparison is represented by Fig 13 and Fig 14 in the term of dropped request probability. First, Fig 13 compares the probability of dropped request for M2M users of DBS algorithm by QoS-RAS one for smart metering use case scenario under different number of UEs. The probabilities of compared schemes increase as the number of UEs increases. QoS-RAS leads to the least dropped probability request, but 
DBS yields the highest one. The reason is that QoS-RAS is not only a HetNet network model deploying 12 femtocells, but also it proposes an adaptive QoSbased M2M priority as shown in stage 2 for smart metering scenario (Fig. 5). Thus notably minimizes the dropped probability of M2M requests. Nevertheless, the other compared approach, even it also prioritizes the M2M users, it steels a macrocell-only system model. Second, Fig 14 evaluates the same metric for $\mathrm{H} 2 \mathrm{H}$ users. To do so, we consider the videoconferencing scenario which prioritizes $\mathrm{H} 2 \mathrm{H}$ traffic. We notice that the performance of our proposed scheme is better either in low or high user numbers.

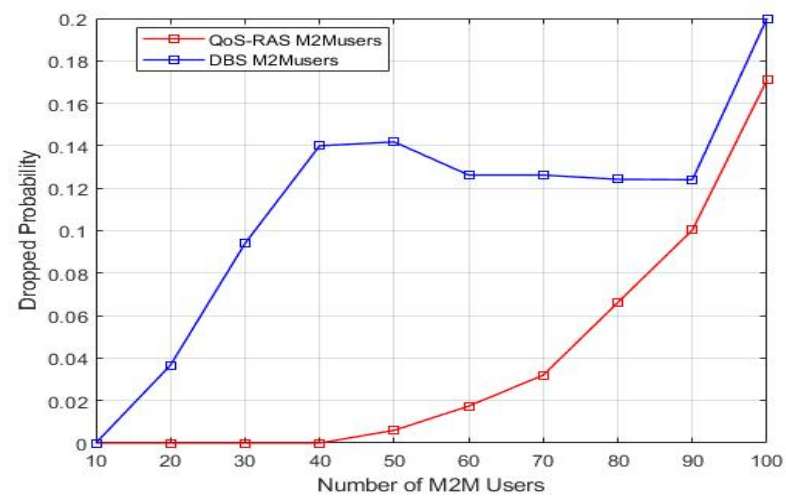

Figure 13: Comparison of the dropped probability of M2M requests

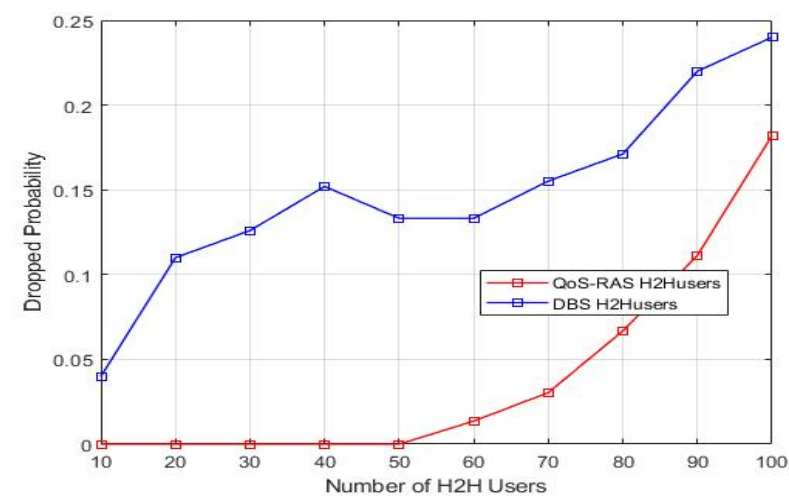

Figure 14: Comparison of the dropped probability of $\mathrm{H} 2 \mathrm{H}$ requests 


\section{Conclusion}

We proposed a QoS-aware resource allocation and femtocell selection for 5G HetNet. Our QoS-RAS scheme aims to enhance system model performances in terms of guaranteeing QoS satisfaction for $5 \mathrm{G}$ specific use cases related to smart metering and videoconferencing scenarios. The objective of our system is to maximize the total resource utilization of the network and to ensure a balanced load by selecting the adequate femtocell for each type of user. Our QoS-RAS ensures the selection of the relevant femtocell by executing the utility function in the first stage. It provides also an adaptive QoS-based priority resource allocation between $\mathrm{H} 2 \mathrm{H}$ and M2M users in the second stage. Since our proposed scheme focuses only on the $\mathrm{M} 2 \mathrm{M}$ and $\mathrm{H} 2 \mathrm{H}$ users, we aim to extend our research considering V2X users and further use cases and application for 5 G HetNets.

\section{References}

[1] Osseiran, A., Braun, V., Hidekazu, T., Marsch, P., Schotten, H., Tullberg, H,\& Schellman, M. (2013, June), The foundation of the mobile and wireless communications system for 2020 and beyond: Challenges, enablers and technology solutions, In Vehicular Technology Conference (VTC Spring), 2013 IEEE 77th (pp. 1-5). IEEE.

[2] Li, Q. C., Niu, H., Papathanassiou, A. T., \& Wu, G. (2014), 5G network capacity: Key elements and technologies, IEEE Vehicular Technology Magazine, 9(1), 71-78.

[3] Araújo, D. C., Maksymyuk, T., de Almeida, A. L., Maciel, T., Mota, J. C., \& Jo, M. (2016), Massive MIMO: survey and future research topics, Iet Communications, 10(15), 1938-1946.

[4] Singh, S., \& Singh, P. (2012), Key concepts and network architecture for 5G mobile technology, International Journal of Scientific Research Engineering 
\& Technology (IJSRET), IIMT Engineering College, Meerut, India, 1(5), 165-170.

[5] https://5g.co.uk/guides/small-cells-hetnets-5g/

[6] Takeda, K., Harada, H., Nagata, S., and Ishii, H. (2016), Small base station, user terminal and radio communication method, U.S. Patent Application No. $15 / 024,983$.

[7] Sun, Y., \& Peng, M. (2018), Recent Advances of Heterogeneous Radio Access Networks: A Survey, Journal of Mobile Multimedia, 14(4), 345-366.

[8] Hasan, M., \& Hossain, E. (2014),Distributed resource allocation in 5 G cellular networks, Towards 5G: Applications, requirements and candidate technologies, 129-161.

[9] Assaad, M. (2014), Resource Allocation Challenges in Future Wireless Networks.

[10] Saddoud, A., Doghri, W., Charfi, E., \& Fourati, L. C. (2020), 5 G radio resource management approach for multi-traffic IoT communications, Computer Networks, 166, 106936.

[11] Saddoud, A., Doghri, W., Charfi, E., \& Fourati, L. C. (2018, September), 5G Radio Resource Management Approach for Internet of Things Communications, In International Conference on Ad-Hoc Networks and Wireless (pp. 77-89). Springer, Cham.

[12] Saddoud, A., Doghri, W., Charfi, E., \& Fourati, L. C. (2019, June), 5G Dynamic Borrowing Scheduler for IoT communications, In 2019 15th International Wireless Communications \& Mobile Computing Conference (IWCMC) (pp. 1630-1635). IEEE.

[13] Jiang, F., Wang, B. C., Sun, C. Y., Liu, Y., \& Wang, X. (2018). Resource allocation and dynamic power control for D2D communication underlaying uplink multi-cell networks, Wireless Networks, 24(2), 549-563. 
[14] Hasabelnaby, M. A., Selmy, H. A., \& Dessouky, M. I. (2019) Optimal Resource Allocation for Cooperative Hybrid FSO/mmW 5G Fronthaul Networks, In 2019 IEEE Photonics Conference (IPC) (pp. 1-2). IEEE.

[15] Bansod, R., Shastry, A., Kumar, B., \& Mishra, P. K. (2018), GA-Based Resource Allocation Scheme for D2D Communcation for $5 G$ Networks, In 2018 International Conference on Inventive Research in Computing Applications (ICIRCA) (pp. 748-752). IEEE.

[16] Zhou, Y., Fadlullah, Z. M., Mao, B., \& Kato, N. (2018). A deep-learningbased radio resource assignment technique for $5 G$ ultra dense networks. IEEE Network, 32(6), 28-34.

[17] M. Elkourdi, A. Mazin and R. D. Gitlin, "Towards Low Latency in 5G HetNets: A Bayesian Cell Selection / User Association Approach," 2018 IEEE 5G World Forum (5GWF), Silicon Valley, CA, 2018, pp. 268-272, doi: 10.1109/5GWF.2018.8517073.

[18] M. F. Khan, "An Approach for Optimal Base Station Selection in 5G HetNets for Smart Factories," 2020 IEEE 21st International Symposium on "A World of Wireless, Mobile and Multimedia Networks" (WoWMoM), Cork, Ireland, 2020, pp. 64-65, doi: 10.1109/WoWMoM49955.2020.00025.

[19] M. Yoshino, H. Shingu, H. Asano, Y. Morihiro and Y. Okumura, "Optimal Cell Selection Method for 5G Heterogeneous Network," 2019 IEEE 89th Vehicular Technology Conference (VTC2019-Spring), Kuala Lumpur, Malaysia, 2019, pp. 1-5, doi: 10.1109/VTCSpring.2019.8746432.

[20] A. Omran, L. Sboui, B. Rong, H. Rutagemwa and M. Kadoch, "Joint Relay Selection and Load Balancing using D2D Communications for 5G HetNet MEC," 2019 IEEE International Conference on Communications Workshops (ICC Workshops), Shanghai, China, 2019, pp. 1-5, doi: 10.1109/ICCW.2019.8756853. 
[21] Wooseong Kim, "Dual Connectivity in Heterogeneous Small Cell Networks with mmWave Backhauls", Mobile Information Systems, vol. 2016, Article ID 3983467, 14 pages, 2016. https://doi.org/10.1155/2016/3983467

[22] Bouaziz, A., Saddoud, A., Fourati, L. C., \& Chaouchi, H. (2018, September). Dynamic Joint Resource Allocation and Femtocell Selection for 5G HetNet. In International Conference on Ad-Hoc Networks and Wireless (pp. 90-101). Springer, Cham. 